\title{
Administration of Umbilical Cord Blood Cells Transiently Decreased Hypoxic-Ischemic Brain Injury in Neonatal Rats
}

\author{
Tetsuo Hattori ${ }^{a}$ b Yoshiaki Sato $^{a}$ Taiki Kondo ${ }^{a}$ Yuko Ichinohashi ${ }^{a}$ \\ Yuichiro Sugiyama ${ }^{a}$ Michiro Yamamoto ${ }^{c}$ Tomomi Kotani $^{d}$ Hitoshi Hirata $^{c}$

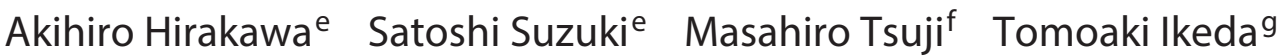 \\ Keiko Nakanishi $^{\text {h }}$ Seiji Kojima ${ }^{b}$ Klas Blomgren ${ }^{i}$ Masahiro Hayakawa ${ }^{a}$ \\ aDivision of Neonatology, Center for Maternal-Neonatal Care, Nagoya University Hospital, Departments of \\ ${ }^{b}$ Pediatrics, ${ }^{\mathrm{C} H a n d}$ Surgery and d Obstetrics and Gynecology, and ${ }^{\mathrm{e} C e n t e r ~ f o r ~ A d v a n c e d ~ M e d i c i n e ~ a n d ~ C l i n i c a l ~}$ \\ Research, Nagoya University Graduate School of Medicine, Nagoya, ${ }^{\mathrm{f}}$ Department of Regenerative Medicine and \\ Tissue Engineering, National Cerebral and Cardiovascular Center Research Institute, Osaka, 9 Department of \\ Obstetrics and Gynecology, Mie University, Mie, and hepartment of Perinatology, Institute for Developmental \\ Research, Aichi Human Service Center, Aichi, Japan; 'Karolinska Institute, Department of Women's and Children's \\ Health, Karolinska University Hospital, Stockholm, Sweden
}

\section{Key Words}

Neuroprotection · Oxidative stress · Cell therapy · Asphyxia

\begin{abstract}
This study aimed to investigate whether the administration of mononuclear cells derived from human umbilical cord blood cells (UCBCs) could ameliorate hypoxic-ischemic brain injury in a neonatal rat model. The left carotid arteries of 7-day-old rats were ligated, and the rats were then exposed to $8 \%$ oxygen for $60 \mathrm{~min}$. Mononuclear cells derived from UCBCs using the Ficoll-Hypaque technique were injected intraperitoneally $6 \mathrm{~h}$ after the insult $\left(1.0 \times 10^{7}\right.$ cells). Twentyfour hours after the insult, the number of cells positive for the oxidative stress markers 4-hydroxy-2-nonenal and nitrotyrosine, in the dentate gyrus of the hippocampus in the UCBC-treated group, decreased by 36 and $42 \%$, respectively, compared with those in the control group. In addition, the number of cells positive for the apoptosis markers active caspase- 3 and apoptosis-inducing factor decreased by 53 and
\end{abstract}

(C) 2015 S. Karger AG, Basel

0378-5866/15/0372-0095\$39.50/0
$58 \%$, respectively. The number of activated microglia (ED1positive cells) was $51 \%$ lower in the UCBC group compared with the control group. In a gait analysis performed 2 weeks after the insult, there were no significant differences among the sham-operated, control and UCBC groups. An active avoidance test using a shuttle box the following week also revealed no significant differences among the groups. Neither the volumes of the hippocampi, corpus callosum and cortices nor the numbers of neurons in the hippocampus were different between the UCBC and control groups. In summary, a single intraperitoneal injection of UCBC-derived mononuclear cells $6 \mathrm{~h}$ after an ischemic insult was associated with a transient reduction in numbers of apoptosis and oxidative stress marker-positive cells, but it did not induce longterm morphological or functional protection. Repeated administration or a combination treatment may be required to achieve sustained protection.

(c) 2015 S. Karger AG, Basel

Tetsuo Hattori and Yoshiaki Sato contributed equally to this work.

\section{KARGER 125}

E-Mail karger@karger.com www.karger.com/dne
Yoshiaki Sato, MD, $\mathrm{PhD}$

Division of Neonatology, Center for Maternal-Neonatal Care Nagoya University Hospital

65 Tsurumai-cho, Showa-ku, Nagoya 466-8560 (Japan)

E-Mail yoshiaki@med.nagoya-u.ac.jp 


\section{Introduction}

Perinatal hypoxia-ischemia (HI) remains an important cause of neonatal death and permanent neurological deficits [1]. Notwithstanding the developments made in perinatal medicine, perinatal HI occurs in 1.3-1.7/1,000 live births; its incidence is high even in developed countries [2]. Many survivors of perinatal HI experience longterm neurological disabilities and impairments resulting in major socioeconomic burdens. At present, there are no effective treatments for HI-induced brain damage, except for brain hypothermia [3], which is not effective in severe cases $[4,5]$. Therefore, it is of the utmost importance to develop a novel and effective therapy against perinatal HI-induced brain injury.

Stem cell therapy is expected to be used in the treatment of many central nervous system diseases in the future. Various kinds of stem cells are possible sources of cell therapy for future clinical applications $[6,7]$. We recently demonstrated that intracerebroventricular injection of neural stem/progenitor cells together with chondroitinase $\mathrm{ABC}$ - which digests glycosaminoglycan chains on chondroitin sulfate proteoglycans - significantly decreased the degree of cerebral infarction after perinatal HI injury in a rat model [8]. However, ethical concerns hinder the use of postmortem human brains as a source of neural stem/progenitor cells in future clinical applications. Furthermore, intracerebral administration is an invasive procedure, and the injected cells themselves may lead to gliotic changes in the host brain [9], thereby necessitating more detailed examinations to ensure the safety of the procedure.

Umbilical cord blood cells (UCBCs) are a promising source of stem cell therapy. They are readily available and can be used for autologous transplantations. Thus, many ethical considerations can be avoided. Furthermore, UCBCs can be administered intravenously [10] and cross the blood-brain barrier [11]. Meier et al. [12] first reported the treatment effects of UCBCs in the amelioration of $\mathrm{HI}$-induced brain damage in a neonatal rat model; moreover, several recent studies reported favorable effects of UCBCs [13-18]. However, the mechanisms underlying the favorable effects remain to be fully elucidated. In the present study, we administered UCBCs to HI rats to investigate their effects and the underlying mechanisms.

\section{Materials and Methods}

Animals

All animal experimental protocols in the present study were approved by the Institutional Review Board of Nagoya University
School of Medicine (Nagoya, Aichi Prefecture, Japan; permit No.: 23181-2011 and 24337-2012). Wistar/ST rat pups were obtained from Japan SLC Inc. (Shizuoka, Japan) and maintained under a 12-hour light/dark cycle (8.00 a.m. to 8.00 p.m.) with ad libitum access to food and water. The animal room and experimental space were always maintained at $23^{\circ} \mathrm{C}$.

\section{UCBC Preparation}

Human UCBCs were donated by women who delivered at $\mathrm{Na}$ goya University Hospital. Written, informed consent was obtained from the donors and their spouses, and this experimental protocol using human cells was reviewed and approved by the local ethics committee of our hospital (permit No.: 794). The donors underwent normal delivery or elective cesarean section because of previous cesarean section, breech position or cephalopelvic disproportion. The donors and infants had no major perinatal complications; all were singleton pregnancies of more than 36 weeks of gestational age.

Umbilical cord blood was collected immediately after placental delivery in bags containing citrate phosphate dextrose (CBC-20; Nipro Corporation, Osaka, Japan). Mononuclear cells were isolated using the Ficoll-Hypaque technique, suspended in Roswell Park Memorial Institute (RPMI) 1640 medium (Life Technologies, Carlsbad, Calif., USA) at a concentration of $1 \times 10^{7}$ cells $/ \mathrm{ml}$, and cryopreserved in liquid nitrogen with an equal amount of a cryoprotectant (CP-1; Kyokuto Pharmaceutical Industrial Co. Ltd., Tokyo, Japan). CP-1 is a mixture of dimethylsulfoxide and hydroxyethyl starch, which makes it possible to preserve stem cells in a frozen state. Immediately before administration, the cells were thawed to $37^{\circ} \mathrm{C}$, washed 3 times with phosphate-buffered saline (PBS) and resuspended in $0.3 \mathrm{ml}$ of RPMI 1640 medium.

\section{HI Insult and UCBC Administration}

$\mathrm{HI}$ brain damage was produced using postnatal day 7 (P7) rats according to the method of Rice et al. [19] with slight modifications. Each pup was anesthetized using isoflurane inhalation and the left carotid artery was subsequently doubly ligated and incised between the ligatures. After a 1-hour rest with a dam, the pups were exposed to a hypoxic environment of $8 \% \mathrm{O}_{2}$ at $37^{\circ} \mathrm{C}$ for $60 \mathrm{~min}$, after which they were returned to the dam in the animal room maintained at $23^{\circ} \mathrm{C}$. Six hours later, the pups in the treatment group (UCBC group) were injected intraperitoneally with mononuclear cells derived from UCBCs $\left(1 \times 10^{7}\right.$ cells $\left./ 0.3 \mathrm{ml}\right)$. A control group underwent ligation of the left carotid artery and hypoxia in the same manner, but received an equivalent volume of RPMI medium alone. The sham group underwent neither left carotid artery ligation nor hypoxia.

\section{Histological and Immunohistochemical Procedures}

Histological and immunohistochemical procedures were performed as previously described [20] with minor modifications. Briefly, rats were deeply anesthetized and intracardially perfusion-fixed with $0.9 \% \mathrm{NaCl}$ followed by $4 \%$ paraformaldehyde in PBS. The brains were removed and immersion-fixed in the same solution at $4{ }^{\circ} \mathrm{C}$ for $24 \mathrm{~h}$, dehydrated with a graded series of ethanol and xylene, embedded in paraffin and cut into $5-\mu \mathrm{m}$-thick coronal sections. After deparaffinization and rehydration, antigen retrieval was performed by heating the sections for $10 \mathrm{~min}$ in $10 \mathrm{~mm}$ citrate buffer ( $\mathrm{pH}$ 6.0). Nonspecific binding was blocked with 3\% donkey serum in PBS. Then, sections were incubated overnight at $4{ }^{\circ} \mathrm{C}$ with rabbit anti-active caspase- 3 (product No. 559565; dilution 1:200; BD Pharmingen, Franklin Lakes, N.J.,
96

Dev Neurosci 2015;37:95-104 DOI: $10.1159 / 000368396$
Hattori et al. 
USA), goat anti-apoptosis-inducing factor (AIF; product No. SC9416; dilution 1:100; Santa Cruz Biotechnology, Dallas, Tex., USA), rabbit anti-4-hydroxy-2-nonenal (4-HNE; product No. HNE11-S; dilution 1:400; Alpha Diagnostic International, San Antonio, Tex., USA), rabbit antinitrotyrosine (product No. A-21285; dilution 1:200; Life Technologies; control samples are shown in the online suppl. fig. 1; for all online suppl. material, see www.karger.com/doi/10.1159/000368396), mouse anti-ED1 (activated microglia marker; product No. MAB1435; dilution 1:300; Merck Millipore), goat anti-neuronal nuclei (NeuN) antibody (product No. MAB377; dilution 1:400; Merck Millipore) or rat anti-myelin basic protein antibody (product No. MAB386; dilution 1:200; Merck Millipore) in PBS with $0.1 \%$ Triton. The sections were subsequently incubated with the appropriate biotinylated secondary antibodies (Vector Laboratories, Burlingame, Calif., USA) for $1 \mathrm{~h}$ at room temperature. Endogenous peroxidase activity was blocked with $3 \% \mathrm{H}_{2} \mathrm{O}_{2}$ in PBS for 10 min and then avidin-biotin-peroxidase complex (Vectastain ABC Elite kit; Vector Laboratories), followed by peroxidase detection for $10 \mathrm{~min}$

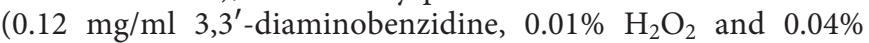
$\mathrm{NiCl}_{2}$ ).

\section{Cell Counting for Acute Injury Markers}

Cells positive for active caspase-3, nuclear AIF, 4-HNE, nitrotyrosine and ED1 were counted throughout the hippocampal granule cell layer (GCL). In the section at the hippocampal level, which is approximately -3.3 to $-3.6 \mathrm{~mm}$ posterior to the bregma in the adult rat brain [21], the GCL was outlined under low magnification $(\times 100)$, and the area was measured. Further, the positive cells were counted under high magnification $(\times 400)$ using Stereo Investigator version 10 stereology software (MicroBrightField Europe EK, Magdeburg, Germany) and expressed as densities.

\section{Behavioral Tests}

Gait Analysis

To evaluate hemiparesis, the CatWalk ${ }^{\circledR}$ quantitative gait analysis system (Noldus Information Technology, Wageningen, the Netherlands) was used for gait assessment following experimental stroke [22]. Briefly, we had the experimental rats run across a glass walkway transversely and recorded the runs using a camera positioned below. If an animal failed to complete a run within $5 \mathrm{~s}$, walked backwards or reared during the run, it was made to repeat the process. Each rat ran 3 times, and the average was calculated. The experiment was performed in the dark; the glass walkway was illuminated with beams of light, thereby allowing the animals' paws to reflect light as they touched the glass floor. An observer labeled each paw on the recorded video to calculate paw-related parameters. The gait-related parameters measured using the CatWalk system were the following: run duration, time duration of entire run; print area, area of paw print; stride length, distance the paw traveled from one step to the next; swing speed, the stride length divided by the duration of one stride length; mean intensity, the mean intensity of each paw in the run (the intensity is proportional to the load on the paw); duty cycle, percentage of time the paw accounted for the total step cycle of that paw.

Active Avoidance Test

The active avoidance test was performed according to the methods of Ichinohashi et al. [23] using the same equipment. For 4 consecutive days, each rat underwent 20 sessions of the active avoidance test each day. The test was conducted in an automated shuttle box (Med Associates Inc., St. Albans, Vt., USA) subdivided into 2 compartments with independently electrified stainless steel bars as a floor. One session consisted of a buzzer tone and stimulation with light (conditioning stimulus), and an electric shock (unconditioned stimulus). The conditioning stimulus was continued for $5 \mathrm{~s}$, as was the subsequent unconditioned stimulus with a positive half-wave constant current of $0.5 \mathrm{~mA}$. When the conditioning stimulus occurred, the rat had to escape to the other side of the shuttle box to turn it off and avoid the unconditioned one. The average interval time between each trial was $30 \mathrm{~s}$ (range, 10-90 s). The parameters were analyzed using the MED-PC IV program (Med Associates Inc.). Each day, we evaluated the avoidance proportion, i.e. the number of sessions in which the rat successfully avoided the unconditioned stimulus.

\section{Volume Measurement and Stereological Cell Counting}

After the behavior tests, the rats were deeply anesthetized, intracardially perfusion fixed, and had their brains removed. To evaluate the whole cortex, corpus callosum and hippocampus, every 150th section from the whole cerebrum and corpus callosum (typically 17 sections) and every 75th section from the hippocampus level (typically 6 sections) were stained using goat anti-NeuN antibody for cortex and hippocampus, or anti-myelin basic protein antibody for the corpus callosum. The bilateral cortex, hippocampus (NeuN-positive areas) and corpus callosum of each section were outlined, and the areas of each were measured using Stereo Investigator. The volumes of the bilateral cortex and hippocampus were calculated from the NeuN-positive areas according to the Cavalieri principle using the following formula: $\mathrm{V}=\Sigma \mathrm{A} \times \mathrm{P} \times \mathrm{T}$, where $\mathrm{V}=$ the total volume, $\Sigma \mathrm{A}=$ the sum of area measurements, $\mathrm{P}=$ the inverse of the sampling fraction, and $\mathrm{T}=$ the section thickness [20]. The numbers of NeuN-positive cells were counted in the whole GCL, including the subgranular zone, of the hippocampus using unbiased stereological counting techniques. After outlining the borders of the GCL, the computer program overlays the outlined area with a grid system of counting frames. Cells within these frames as well as those touching 2 out of 4 predetermined sides of the frames were counted.

\section{Statistical Analysis}

All data are presented as mean \pm standard error of the mean. A 2 -sample Student's t test was used to compare the two groups in figure 2. The Steel-Dwass test as nonparametric multiple comparison procedure was used to compare the three groups in figures 3 and 4 . A p value $<0.05$ was considered statistically significant.

\section{Results}

\section{Impact of UCBCs on Expression of Acute Injury}

Markers after $H I$

P7 rats were subjected to HI, and $6 \mathrm{~h}$ later, they were administered cryopreserved, thawed and washed mononuclear cells isolated from the UCBCs or vehicle. We examined various acute injury biomarkers $24 \mathrm{~h}$ after $\mathrm{HI}$ : apoptosis markers (active caspase-3 and AIF), oxidative 
Fig. 1. Photomicrographs of the hippocampus stained for acute injury markers. Representative photomicrographs of the dentate gyrus of the hippocampus $24 \mathrm{~h}$ after $\mathrm{HI}$ insult. The sections from vehicle-treated rats (vehicle) and UCBC-treated rats (UCBC) were stained for active caspase-3 (a), AIF (b), 4-HNE (c), nitrotyrosine (d) and $\operatorname{ED} 1(\mathbf{e})$. Bar $=100 \mu \mathrm{m}$. Insets show higher magnifications.

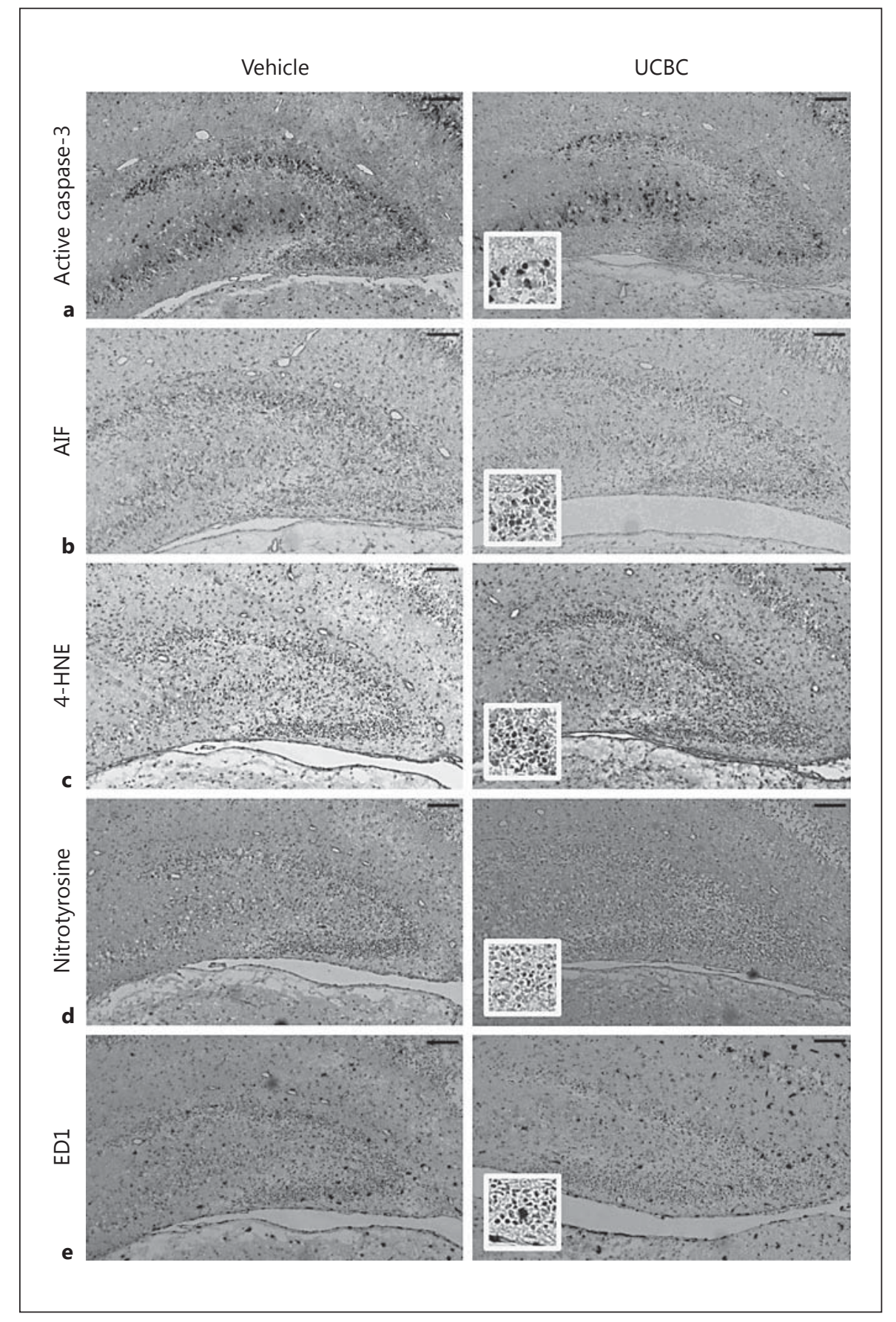

stress markers (4-HNE and nitrotyrosine) and an activated microglia marker (ED1). Photomicrographs of representative hippocampi are shown in figure 1. The number of the apoptosis marker-positive cells in the ipsilateral GCL significantly decreased in the UCBC group compared with those in the control group (activated caspase- $3,53 \%$, and AIF, 58\%; fig. 2a, b; p $<0.05$ and $p<$
0.01 , respectively). The numbers of oxidative stress marker-positive cells also decreased in the UCBC group compared with the control group (4-HNE, 36\%, and nitrotyrosine, $42 \%$; fig. $2 \mathrm{c}, \mathrm{d}$; $\mathrm{p}<0.05)$. Moreover, the number of ED1-positive cells was 51\% lower in the UCBC group compared with the control group (fig. 2e; $\mathrm{p}<0.05)$. 


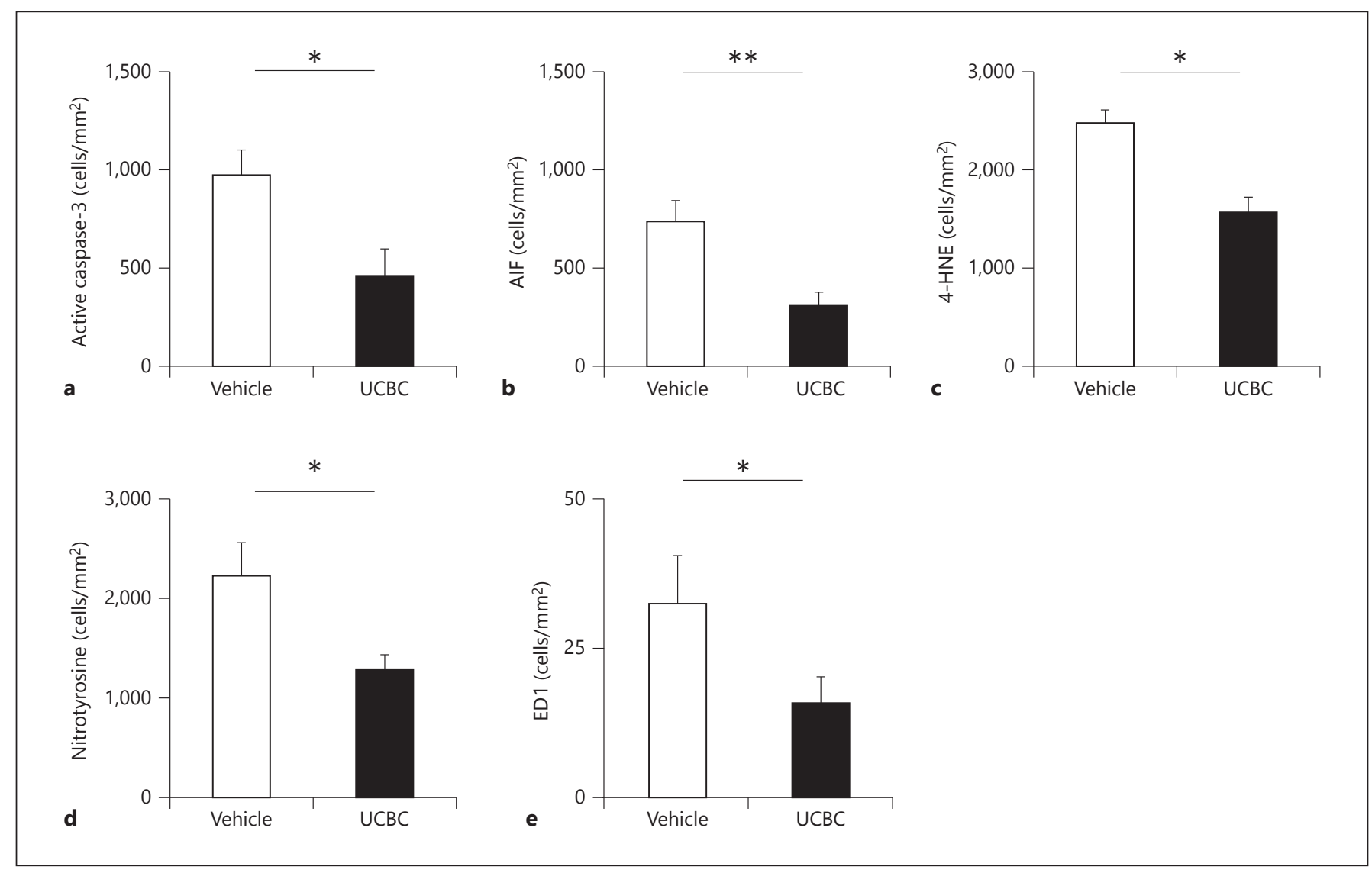

Fig. 2. Impact of UCBCs on apoptosis, oxidative stress and activation of microglia. The numbers of various marker-positive cells in figure 1 were counted. The numbers of active caspase-3-positive (a) and AIF-positive cells (b) in the ipsilateral GCL were significantly lower in the UCBC group $(n=11)$ than in the vehicle group $(\mathrm{n}=12)$. The numbers of both 4-HNE- (c) and nitrotyrosine-positive cells (d) were also significantly lower in the UCBC group. The number of ED1-positive cells (e) was significantly decreased in the UCBC group. Data are presented as means \pm standard error of the mean. ${ }^{*} \mathrm{p}<0.05,{ }^{* *} \mathrm{p}<0.01$.

\section{Impact of UCBCs on Behavior after HI Gait Analysis}

To evaluate the effect of HI on motor function and of UCBC administration on HI-induced motor deficits, gait analysis was performed 14 days after the insult (P21) using the CatWalk system. There were no significant differences in run duration, right front paw (RF) print area, $\mathrm{RF}$ swing speed, $\mathrm{RF} /$ left front paw (LF) ratio of mean intensity, RF duty cycle or RF stride length among the three groups (sham-operated, control and UCBC groups; fig. 3).

\section{Active Avoidance Test}

Further, to evaluate the effects of UCBCs on HI-induced learning impairments, an active avoidance test was performed 21-24 days after the insult (P28-31). The mean avoidance proportion of each group was calculated for each consecutive day (fig. 4). The avoidance rates increased with time in all groups; however, there were no significant differences among the three groups for each day.

\section{Impact of UCBCs on Histological Changes after HI}

Finally, to assess the absolute tissue loss after HI, the volumes of the cortex, corpus callosum and hippocampus of both hemispheres were evaluated. After the behavioral tests, sections throughout the whole cerebrum were evaluated.

Representative photomicrographs stained for NeuN are shown in figure $5 \mathrm{a}$ and $\mathrm{b}$. In both groups, there was apparent unilateral atrophy with partial collapse. We evaluated the volumes of the cortex (fig. 5c), corpus callosum (fig. 5d) or hippocampus (fig. 5e), but found no significant differences in the volumes between the groups. 


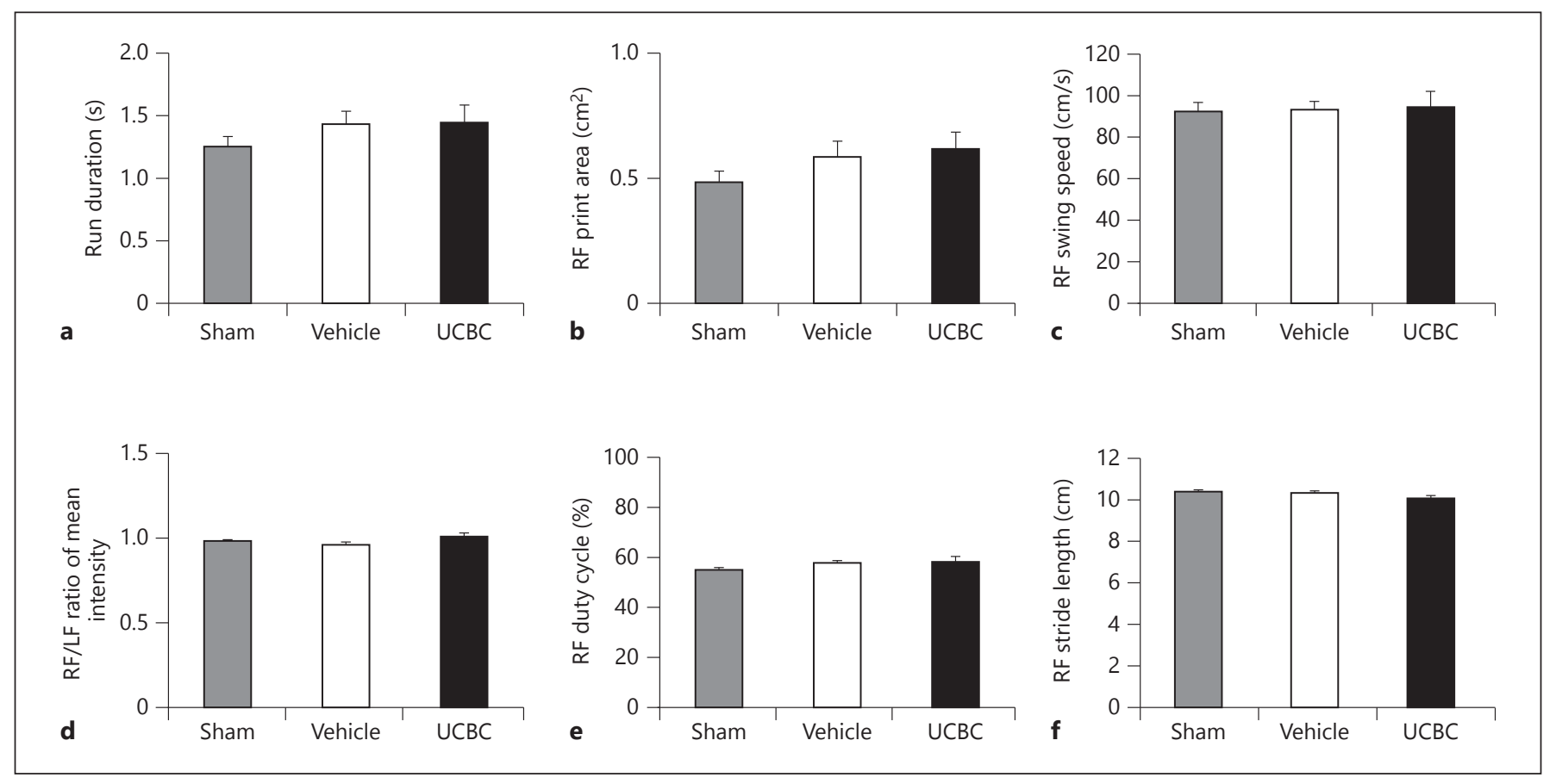

Fig. 3. Gait analysis. Gait analysis was performed 2 weeks after the insult. Each parameter was compared among the groups. There was no significant difference in run duration (a), RF print area (b),
RF swing speed (c), RF/LF ratio of mean intensity (d), RF duty cycle (e) or RF stride length (f) among the three groups (shamoperated, $n=6$; vehicle, $n=8$; UCBC, $n=6$ ).

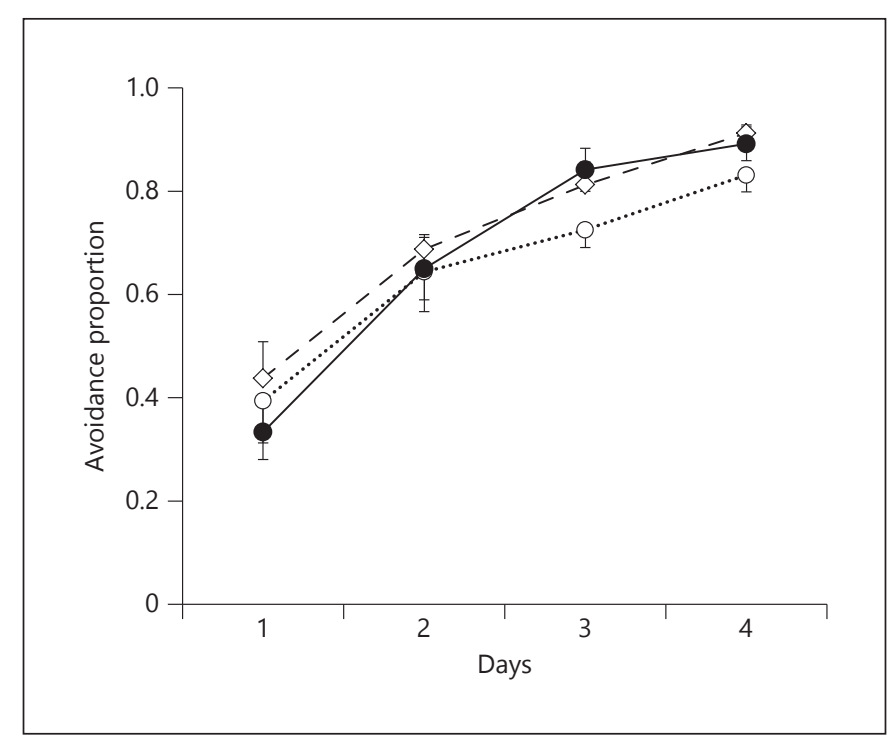

Fig. 4. Active avoidance test. The active avoidance was performed 21-24 days after insult (P28-31). Each rat underwent 20 sessions every day, and the mean avoidance proportion was calculated for each group. The avoidance proportions increased with time in all groups. There was no significant difference in avoidance proportions among the groups on any day. Open squares and dashed line: sham-operated, $\mathrm{n}=4$; open circles and dotted line: vehicle, $\mathrm{n}=8$; closed circles and solid line: $\mathrm{UCBC}, \mathrm{n}=6$.
Therefore, we examined whether there was a difference in the number of neurons between the groups, even though the volume reductions were equivalent. The numbers of NeuN-positive neurons in the hippocampus were counted using stereological principles (Stereo Investigator, MicroBrightField) but they were not significantly different between the groups in the hippocampus of either the ipsilateral or contralateral hemisphere (fig. 5f).

\section{Discussion}

In the present study, we demonstrated that intraperitoneal UCBC administration caused antiapoptotic and antioxidative effects $24 \mathrm{~h}$ after the insults; however, we failed to demonstrate a prolonged reduction of neurological damage. We administered UCBCs in the early phase ( $6 \mathrm{~h}$ after $\mathrm{HI}$ ). The optimal timing of administration is one of the most critical points to establish a new cell therapy. In regenerative medicine using stem cells, grafting in the early postinjury phase is generally not recommended. In neural stem/progenitor cell transplantation, early grafting in the acute phase (i.e. $24 \mathrm{~h}$ after insult), during which inflammatory chemical mediator and 
Fig. 5. Impact of the UCBCs on the histological change after HI. The volumes of whole cortex, corpus callosum and hippocampus, and the number of NeuN-positive neurons in whole hippocampus were evaluated using Stereo Investigator version 10 (stereology software) after behavioral tests. a, b Representative photomicrographs of the brain stained for NeuN from vehicle (a) and UCBC-treated rats (b) 2 months after HI. Bar $=1,000 \mu \mathrm{m}$. There was no significant difference in the volumes of the cortex (c), corpus callosum (d) or hippocampus (e) between the groups. The numbers of NeuN-positive neurons in the GCL were not significantly different between the vehicle and UCBC groups in the hippocampus of ipsilateral hemispheres (f). Contra. = Contralateral; Ipsi. = ipsilateral.

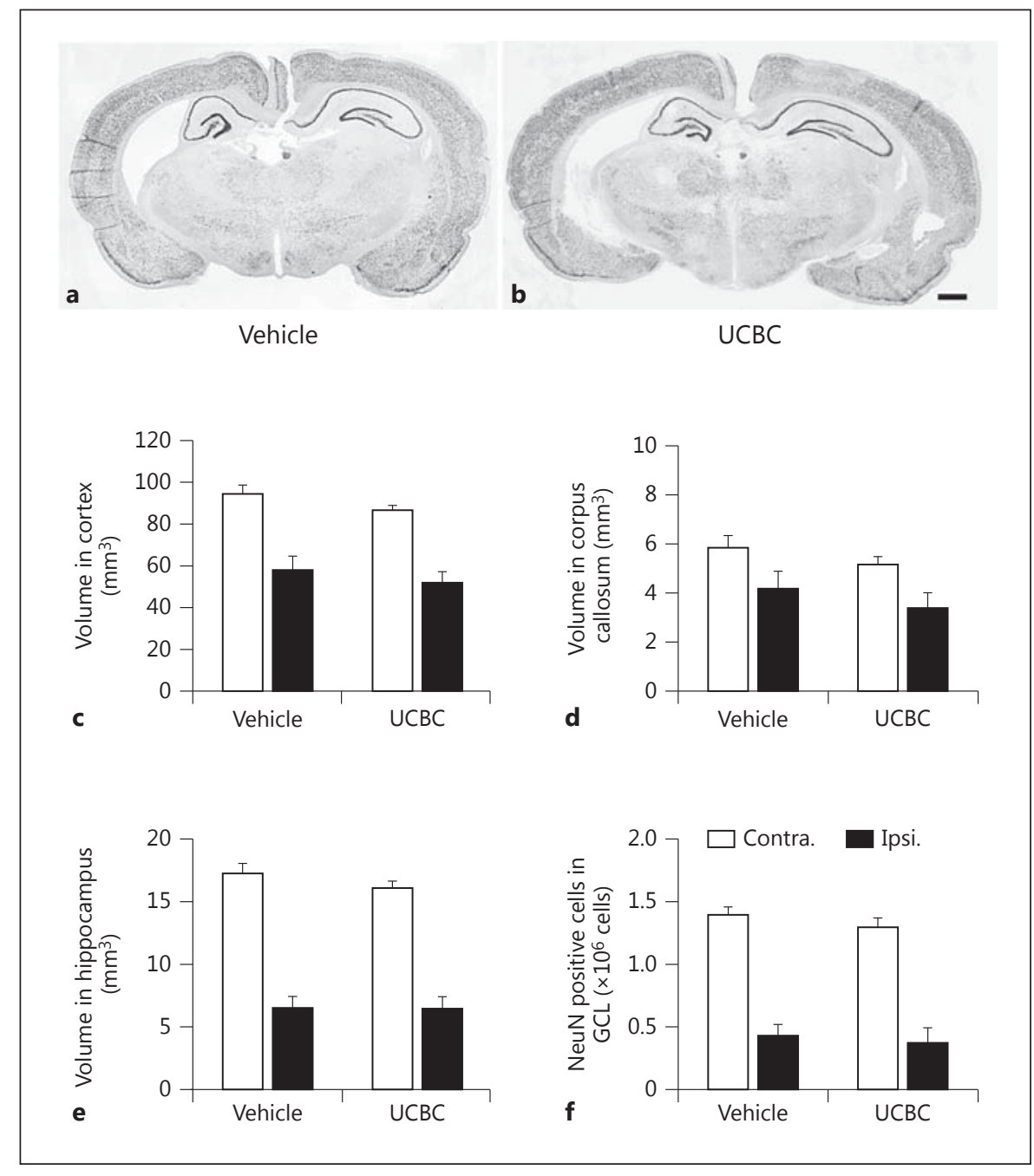

cytokine concentrations are increased [24], can induce greater differentiation of grafted cells to astrocytes, decrease the survival rates and/or decrease the beneficial treatment effects in various models such as spinal cord injury [25] and stroke [26] in adult rodents, and irradiation-induced brain injury in young mice [9]. In addition, early administration of mononuclear cells from bone marrow showed fewer treatment effects in a model of adult stroke [27]. However, in neonatal HI, there are two phases of pathological events: primary and secondary energy failure $[28,29]$. Primary energy failure occurs within minutes after initial cerebral ischemia; in this phase, acidosis and depletion of oxygen, glucose and adenosine triphosphate lead to acute derangement of intracellular metabolism, resulting in necrosis and cell death. The subsequent secondary energy failure occurs after a variable period following the initial insult. Inflammation, excit- atory amino acid release, intracellular calcium inflow, and production of nitric oxide and reactive oxidative species occur in this stage. Therefore, it is reasonable that most therapies, including hypothermia therapy, should be commenced at least before the second phase. The treatment effect induced by UCBC administration for brain injury is considered to be involved in secretion of neurotrophic factors that promote endogenous neurogenesis, prevent loss of neuronal cells and regulate immunity [28], which was shown also in the effect of bone marrow-derived mesenchymal stem cells [30]. Expression levels of various pro-inflammatory cytokines, including interleukin-1 and tumor necrosis factor, are elevated in the early phase of perinatal HI; UCBC administration can decrease these levels, which are also accompanied by decreased expression of cluster of differentiation 68 , a biomarker of activated microglia/macrophages in the brain 
[31]. Our study showed a decrease in the number of activated microglia by UCBC administration in the early phase of neonatal HI (fig. 1e, 2e). Pimentel-Coelho et al. [13] also reported that intraperitoneal administration of UCBCs $3 \mathrm{~h}$ after insult decreased apoptosis and microglial activation, and improved primitive reflexes in a neonatal HI rat model. Therefore, to suppress the elevation of such proinflammatory cytokines, which lead to decreased apoptosis, UCBC administration in the early phase may be a reasonable therapeutic approach, as demonstrated in the present study.

Oxidative stress plays an important role in $\mathrm{HI}$ brain damage [32]. Here, we demonstrated a decrease in 4-HNEand nitrotyrosine-positive cells in the dentate gyrus following UCBC administration (fig. 1c, d, 2c, d). To our knowledge, this study is the first to report antioxidative effects of UCBCs in a neonatal HI rat model. Arien-Zakay et al. [33] reported antioxidative effects of UCBC-derived neural progenitor cells on insulted PC12 cell lines. Further, suppression of oxidative stress after adult transient focal ischemia was observed in an interleukin-1 knockout murine model [34]. As described above, UCBC administration can decrease the elevated expression of proinflammatory cytokines including interleukin-1 [31]. Moreover, the decreased expression of ED1 in the present study (fig. 1e, 2e) indicates that UCBC administration decreased HI-induced inflammation. Thus, the antioxidative effect of UCBC administration might be exerted directly and/or via suppression of inflammation.

Calculations of the immunohistochemically stained cells were focused on the dentate gyrus of the hippocampus, which is one of the most vulnerable areas to hypoxic ischemic insult. Although we calculated the immunohistochemically stained cells with density counts, which is less sensitive than stereological counts, the results revealed that $\mathrm{UCBC}$ administration suppressed apoptosis, as indicated by the decrease in the number of cells positive for active caspase- 3 and AIF (fig. 1a, b, 2a, b). In the present study, we performed high-resolution analyses of walking patterns using the CatWalk system; however, they were not sensitive enough to detect motor impairment after $\mathrm{HI}$ injury. In contrast to human neonates, rat pups after HI injury did not show obvious locomotor abnormalities, as in other studies using the same model [35]; this may have been because of the higher degree of plasticity of the immature rat brain [36]. We also evaluated the learning memory after $\mathrm{HI}$ with the shuttle avoidance test and found only a mild tendency to improve the learning memory in the UCBC group; the difference was not significant. This may be a type 2 error, and further studies are required to clarify the potential effects of UCBC therapy on motor impairment and cognitive deficits after HI. In addition, we found no differences between the vehicle and UCBC groups in absolute tissue loss or the number of neurons in the cortex, corpus callosum or hippocampus (fig. 5). Similarly, some former studies failed to show histological improvement following UCBC therapy $[12,16]$, whereas others did $[14,15]$. We have summarized the experimental protocols and results [37]. In many previous reports, $1 \times 10^{7}$ mononuclear cells were administered intraperitoneally $24 \mathrm{~h}$ after the insult. We administered the same dose of cells at an earlier time point. Pimentel-Coelho et al. [13] administered UCBCs even earlier ( $3 \mathrm{~h}$ after the insult) using a lower dose $\left(2 \times 10^{6}\right)$ of cells, and showed improvement in morphology and behavior. It is still unclear how the differences between protocols can affect the results. Other possible reasons for the different outcomes can be the severity of the insult and other experimental settings. Considering the fact that the present study failed to show any effect on morphological changes in the chronic phase or improvement of behavioral impairments, despite the fact that several acute injury markers were suppressed, a modified protocol (e.g. repeated administration, combination with some other treatments) should be tried with an aim to achieve sustained neuroprotection.

In the present study, cryopreserved mononuclear cells were used. Even frozen-thawed UCBCs are known to produce various cytokines and chemokines [38], and exert a neuroprotective effect in various animal models [13, 37, 39]. Moreover, from the viewpoint of clinical applications, cryopreservation is essential in the case of allogeneic transplantation, which may be applicable for patients without access to their own cord blood cells.

We used human UCBCs in a rodent injury model. It might have been more suitable to use rat UCBCs. However, it was very difficult to get sufficient numbers of cells from the umbilical cord of rats without expansion in culture. Because the purpose of the present study was to evaluate the treatment effect of mononuclear cells from the umbilical cord without using culture procedures, we used human cells, as in previous publications [13-18].

Another possible limitation in the present study is that we could not monitor/control body temperature in each pup. We placed the pups on/into the temperature-controlled plate/chamber during the $\mathrm{HI}$ insult, and returned them to the dam in a temperature-controlled room after the insult. There might be some variation in brain temperature, leading to variation in the degree of brain damage [40]. 
The choice of injection site is an important issue when using cell infusion for the treatment of brain injury. We administered UCBCs intraperitoneally, as in most previous studies. According to our recent unpublished results, only a small number of cells either injected intraperitoneally or intravenously could be found in the brain, and cells injected intraperitoneally were less seen in the liver, lung or spleen than cells injected intravenously, indicating that many intraperitoneally injected cells might have stayed in the peritoneal cavity. The treatment effect might be through trophic factors secreted by the cells [41]. The extent of brain damage can be influenced by the peripheral inflammatory response [42]. Modulating peripheral inflammation can be a therapeutic target. In a traumatic brain injury model, multipotent adult progenitor cells exerted a neuroprotective effect through interaction with resident splenocytes [43].

In summary, these results indicate that a single intraperitoneal injection of UCBC-derived mononuclear cells
$6 \mathrm{~h}$ after HI reduced caspase-3, AIF, microglial activations and oxidative stress, but it did not induce morphological or functional protection. Repeated administration or a combination treatment may be required to achieve sustained protection.

\section{Acknowledgements}

This work was supported by JSPS KAKENHI (grant No.: 21890100).

We are grateful to Dr. Atsuhiko Oohira for the helpful discussions and to Ms. Kimi Watanabe, Ms. Eiko Aoki and Ms. Tokiko Nishino for the skillful technical assistance.

\section{Disclosure Statement}

None of the authors have any conflicts of interest associated with this study.

\section{References}

1 Lawn JE, Cousens S, Zupan J: 4 million neonatal deaths: when? Where? Why? Lancet 2005;365:891-900.

2 Kurinczuk JJ, White-Koning M, Badawi N: Epidemiology of neonatal encephalopathy and hypoxic-ischaemic encephalopathy. Early Hum Dev 2010;86:329-338.

-3 Askalan R, Wang C, Shi H, Armstrong E, Yager JY: The effect of postischemic hypothermia on apoptotic cell death in the neonatal rat brain. Dev Neurosci 2011;33:320-329.

4 Azzopardi DV, Strohm B, Edwards AD, Dyet L, Halliday HL, Juszczak E, Kapellou O, Levene M, Marlow N, Porter E, Thoresen M, Whitelaw A, Brocklehurst P: Moderate hypothermia to treat perinatal asphyxial encephalopathy. N Engl J Med 2009;361:1349-1358.

5 Gluckman PD, Wyatt JS, Azzopardi D, Ballard R, Edwards AD, Ferriero DM, Polin RA, Robertson CM, Thoresen M, Whitelaw A, Gunn AJ: Selective head cooling with mild systemic hypothermia after neonatal encephalopathy: multicentre randomised trial. Lancet 2005;365:663-670.

6 Sato Y, Oohira A: Chondroitin sulfate, a major niche substance of neural stem cells, and cell transplantation therapy of neurodegeneration combined with niche modification. Curr Stem Cell Res Ther 2009;4:200-209.

7 Lindvall O, Kokaia Z: Stem cells for the treatment of neurological disorders. Nature 2006; 441:1094-1096.

8 Sato Y, Nakanishi K, Hayakawa M, Kakizawa H, Saito A, Kuroda Y, Ida M, Tokita Y, Aono S, Matsui F, Kojima S, Oohira A: Reduction of brain injury in neonatal hypoxic-ischemic rats by intracerebroventricular injection of neural stem/progenitor cells together with chondroitinase ABC. Reprod Sci 2008; 15 : 613-620.

-9 Sato Y, Shinjyo N, Sato M, Osato K, Zhu C, Pekna M, Kuhn HG, Blomgren K: Grafting of neural stem and progenitor cells to the hippocampus of young, irradiated mice causes gliosis and disrupts the granule cell layer. Cell Death Dis 2013;4:e591.

10 Chen J, Sanberg PR, Li Y, Wang L, Lu M, Willing AE, Sanchez-Ramos J, Chopp M: Intravenous administration of human umbilical cord blood reduces behavioral deficits after stroke in rats. Stroke 2001;32:2682-2688.

11 Garbuzova-Davis S, Willing AE, Zigova T, Saporta S, Justen EB, Lane JC, Hudson JE, Chen N, Davis CD, Sanberg PR: Intravenous administration of human umbilical cord blood cells in a mouse model of amyotrophic lateral sclerosis: Distribution, migration, and differentiation. J Hematother Stem Cell Res 2003; 12:255-270.

-12 Meier C, Middelanis J, Wasielewski B, Neuhoff S, Roth-Haerer A, Gantert M, Dinse HR, Dermietzel R, Jensen A: Spastic paresis after perinatal brain damage in rats is reduced by human cord blood mononuclear cells. Pediatr Res 2006;59:244-249.

13 Pimentel-Coelho PM, Magalhaes ES, Lopes LM, de Azevedo LC, Santiago MF, MendezOtero R: Human cord blood transplantation in a neonatal rat model of hypoxic-ischemic brain damage: functional outcome related to neuroprotection in the striatum. Stem Cells Dev 2010;19:351-358.
14 Wasielewski B, Jensen A, Roth-Harer A, Dermietzel R, Meier C: Neuroglial activation and CX43 expression are reduced upon transplantation of human umbilical cord blood cells after perinatal hypoxic-ischemic injury. Brain Res 2012;1487:39-53.

-15 De Paula S, Greggio S, Marinowic DR, Machado DC, Da Costa JC: The dose-response effect of acute intravenous transplantation of human umbilical cord blood cells on brain damage and spatial memory deficits in neonatal hypoxia-ischemia. Neuroscience 2012; 210:431-441.

16 Geissler M, Dinse HR, Neuhoff S, Kreikemeier K, Meier C: Human umbilical cord blood cells restore brain damage induced changes in rat somatosensory cortex. PLoS One 2011; 6:e20194.

$\checkmark 17$ Rosenkranz K, Kumbruch S, Tenbusch M, Marcus K, Marschner K, Dermietzel R, Meier C: Transplantation of human umbilical cord blood cells mediated beneficial effects on apoptosis, angiogenesis and neuronal survival after hypoxic-ischemic brain injury in rats. Cell Tissue Res 2012;348: 429-438.

18 Yasuhara T, Hara K, Maki M, Xu L, Yu G, Ali MM, Masuda T, Yu SJ, Bae EK, Hayashi T, Matsukawa N, Kaneko Y, Kuzmin-Nichols N, Ellovitch S, Cruz EL, Klasko SK, Sanberg CD, Sanberg PR, Borlongan CV: Mannitol facilitates neurotrophic factor up-regulation and behavioural recovery in neonatal hypoxicischaemic rats with human umbilical cord blood grafts. J Cell Mol Med 2010;14:914921
UCBCs Transiently Decreased HI Brain Injury in Neonatal Rats 
19 Rice JE 3rd, Vannucci RC, Brierley JB: The influence of immaturity on hypoxic-ischemic brain damage in the rat. Ann Neurol 1981;9: 131-141.

20 Osato K, Sato Y, Ochiishi T, Osato A, Zhu C, Sato M, Swanpalmer J, Modjtahedi N, Kroemer G, Kuhn HG, Blomgren K: Apoptosisinducing factor deficiency decreases the proliferation rate and protects the subventricular zone against ionizing radiation. Cell Death Dis 2010;1:e84.

21 Paxinous G, Watson C: The Rat Brain in Stereotaxic Coordinates, ed 2. San Diego, Academic Press, 1986.

22 Encarnacion A, Horie N, Keren-Gill H, Bliss TM, Steinberg GK, Shamloo M: Long-term behavioral assessment of function in an experimental model for ischemic stroke. J Neurosci Methods 2011;196:247-257.

23 Ichinohashi Y, Sato Y, Saito A, Ito M, Watanabe K, Hayakawa M, Nakanishi K, Wakatsuki A, Oohira A: Dexamethasone administration to the neonatal rat results in neurological dysfunction at the juvenile stage even at low doses. Early Hum Dev 2013;89:283288.

24 Nakamura M, Houghtling RA, MacArthur L, Bayer BM, Bregman BS: Differences in cytokine gene expression profile between acute and secondary injury in adult rat spinal cord. Exp Neurol 2003;184:313-325.

25 Okada S, Ishii K, Yamane J, Iwanami A, Ikegami T, Katoh $\mathrm{H}$, Iwamoto Y, Nakamura M, Miyoshi H, Okano HJ, Contag CH, Toyama $\mathrm{Y}$, Okano H: In vivo imaging of engrafted neural stem cells: its application in evaluating the optimal timing of transplantation for spinal cord injury. FASEB J 2005;19:18391841.

26 Rosenblum S, Wang N, Smith TN, Pendharkar AV, Chua JY, Birk H, Guzman R: Timing of intra-arterial neural stem cell transplantation after hypoxia-ischemia influences cell engraftment, survival, and differentiation. Stroke 2012;43:1624-1631.

-27 Uemura M, Kasahara Y, Nagatsuka K, Taguchi A: Cell-based therapy to promote angiogenesis in the brain following ischemic damage. Curr Vasc Pharmacol 2012;10:285-288.
28 Liao Y, Cotten M, Tan S, Kurtzberg J, Cairo MS: Rescuing the neonatal brain from hypoxic injury with autologous cord blood. Bone Marrow Transplant 2013;48:890-900.

29 Lorek A, Takei Y, Cady EB, Wyatt JS, Penrice J, Edwards AD, Peebles D, Wylezinska M, Owen-Reece H, Kirkbride V, et al: Delayed ('secondary') cerebral energy failure after acute hypoxia-ischemia in the newborn piglet: continuous 48-hour studies by phosphorus magnetic resonance spectroscopy. Pediatr Res 1994;36:699-706.

30 Van Velthoven CT, Kavelaars A, van Bel F, Heijnen CJ: Repeated mesenchymal stem cell treatment after neonatal hypoxia-ischemia has distinct effects on formation and maturation of new neurons and oligodendrocytes leading to restoration of damage, corticospinal motor tract activity, and sensorimotor function. J Neurosci 2010;30:9603-9611.

-31 Rosenkranz K, Tenbusch M, May C, Marcus $\mathrm{K}$, Meier C: Changes in interleukin-1 alpha serum levels after transplantation of umbilical cord blood cells in a model of perinatal hypoxic-ischemic brain damage. Ann Anat 2013;195:122-127.

32 Warner DS, Sheng H, Batinic-Haberle I: Oxidants, antioxidants and the ischemic brain. J Exp Biol 2004;207:3221-3231.

33 Arien-Zakay H, Lecht S, Bercu MM, Tabakman R, Kohen R, Galski H, Nagler A, Lazarovici P: Neuroprotection by cord blood neural progenitors involves antioxidants, neurotrophic and angiogenic factors. Exp Neurol 2009;216:83-94.

- 34 Ohtaki H, Takaki A, Yin L, Dohi K, Nakamachi T, Matsunaga M, Horai R, Asano M, Iwakura Y, Shioda S: Suppression of oxidative stress after transient focal ischemia in interleukin-1 knock out mice. Acta Neurochir Suppl 2003;86:191-194.
35 De Paula S, Vitola AS, Greggio S, de Paula D, Mello PB, Lubianca JM, Xavier LL, Fiori HH, Dacosta JC: Hemispheric brain injury and behavioral deficits induced by severe neonatal hypoxia-ischemia in rats are not attenuated by intravenous administration of human umbilical cord blood cells. Pediatr Res 2009;65:631635.

36 Yager JY, Wright S, Armstrong EA, Jahraus CM, Saucier DM: The influence of aging on recovery following ischemic brain damage. Behav Brain Res 2006;173:171-180.

37 Tsuji M, Taguchi A, Ohshima M, Kasahara Y, Sato Y, Tsuda H, Otani K, Yamahara K, Ihara M, Harada-Shiba M, Ikeda T, Matsuyama T: Effects of intravenous administration of umbilical cord blood CD34 cells in a mouse model of neonatal stroke. Neuroscience 2014; 263C:148-158.

- 38 Newman MB, Willing AE, Manresa JJ, Sanberg CD, Sanberg PR: Cytokines produced by cultured human umbilical cord blood (HUCB) cells: implications for brain repair. Exp Neurol 2006;199:201-208.

39 Saporta S, Kim JJ, Willing AE, Fu ES, Davis CD, Sanberg PR: Human umbilical cord blood stem cells infusion in spinal cord injury: engraftment and beneficial influence on behavior. J Hematother Stem Cell Res 2003;12:271-278.

40 DeBow SB, Clark DL, MacLellan CL, Colbourne F: Incomplete assessment of experimental cytoprotectants in rodent ischemia studies. Can J Neurol Sci 2003;30:368-374.

41 Fan CG, Zhang QJ, Tang FW, Han ZB, Wang GS, Han ZC: Human umbilical cord blood cells express neurotrophic factors. Neurosci Lett 2005;380:322-325.

42 Bonestroo HJ, Nijboer CH, van Velthoven CT, Kavelaars A, Hack CE, van Bel F, Heijnen CJ: Cerebral and hepatic inflammatory response after neonatal hypoxia-ischemia in newborn rats. Dev Neurosci 2013;35:197-211.

-43 Walker PA, Shah SK, Jimenez F, Gerber MH, Xue H, Cutrone R, Hamilton JA, Mays RW, Deans R, Pati S, Dash PK, Cox CS Jr: Intravenous multipotent adult progenitor cell therapy for traumatic brain injury: preserving the blood brain barrier via an interaction with splenocytes. Exp Neurol 2010;225:341-352. 